\title{
On curing the divergences in the quark number susceptibility
}

\author{
Rajiv V. Gavai ${ }^{* \dagger}$ \\ Department of Theoretical Physics, Tata Institute of Fundamental Research, \\ Homi Bhabha Road, Mumbai 400005, India. \\ E-mail: gavaietifr.res.in \\ Sayantan Sharma \\ Fakultät für Physik, Universität Bielefeld, \\ D-33615 Bielefeld, Germany \\ E-mail: sayantan@physik.uni-bielefeld.de
}

\begin{abstract}
Adding chemical potential $\mu$ linearly as $\mu N$ to the lattice QCD action, where $N$ is a conserved quark/baryon number, leads to a quadratic divergence as $a^{-2}$. We argue that it is inherited from the continuum theory and can be subtracted off on the lattice following a similar manner in the continuum. We test this idea for quenched quark number susceptibilities and demonstrate a finite continuum limit numerically.
\end{abstract}

The 32nd International Symposium on Lattice Field Theory,

23-28 June, 2014

Columbia University New York, NY

\footnotetext{
* Speaker.

${ }^{\dagger}$ Sir J.C. Bose National Fellow.

$\ddagger$ Currently at Physics Department, Brookhaven National Laboratory, Upton, NY11973
} 


\section{Introduction}

The quark number susceptibilities are important measurables on the lattice due to their relation to the fluctuations of net charge or baryon number that are being measured in the heavy ion collision experiments at RHIC at Brookhaven National Laboratory. Apart from providing information about the degrees of freedom and the interactions of the hot and dense QCD medium, these are also important in estimating the location of the critical point in the QCD phase diagram. The critical point is defined by the singularity of the baryon number susceptibility. Using successive derivatives of the pressure with $\mu_{B} / T$, its location maybe determined by the radius of convergence of the Taylor expansion for it. The radius of convergence depends on the ratios of the higher order quark number susceptibilities, so their accurate and efficient measurement is a challenging problem on the lattice. In order to look for an economic method of computing them, we revisit the problem of introducing chemical potential on the lattice.

As in the continuum, the canonical and most simple way of introducing chemical potential, $\mu$ is by adding a term $\mu N$ to the QCD Lagrangian where $N$ is the charge corresponding to the conserved point-split current on the lattice. It amounts to introducing weights $f(a \mu)=1+a \mu \&$ $g(a \mu)=1-a \mu$ to forward and backward time links in the Dirac operator respectively. However this leads to $\mu$-dependent $a^{-2}$ divergences in energy density and quark number density. To cure this on the lattice one of the popular method $[1,2]$ is to modify the weights to $\exp ( \pm a \mu)$ to obtain

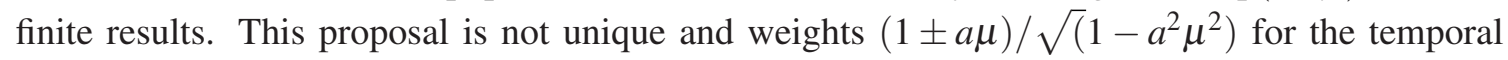
links also lead to finite results [3]. In fact, all that is needed is that any general weights $f, g$ should follow $f(a \mu) \cdot g(a \mu)=1$ with $f(0)=f^{\prime}(0)=1$ in order to cure the undesired divergences on the lattice [4]. It is worth emphasizing here that the analytical proof in all the cases above was for free quarks. Indeed, further numerical computations in quenched QCD showed it to work for the interacting case as well [5], while a similar check in the full theory is still lacking.

Due to the remnant chiral symmetry the staggered quarks possess, a majority of the numerical computations have so far been performed employing them; the chiral symmetry issue gets even more complex for the Wilson fermions. As is well-known, the Overlap and/or Domain Wall Fermions are much more preferable from the chiral symmetry perspective. They have both the correct chiral and flavour symmetry on lattice as well as an index theorem on the lattice [6,7]. These are likely to be crucial for investigations of the QCD critical point, which model-based considerations show to result if QCD has only two light quarks and the axial $U_{A}(1)$ symmetry-restoration takes place at sufficiently large temperatures. A moderately heavy strange quark may affect the location of the QCD critical point but not its existence.

Non-locality of the overlap fermions makes the introduction of the chemical potential as a Lagrange multiplier of the conserved charge nontrivial. Bloch and Wettig [8] proposed to use the same prescription as above for the timelike links of the Wilson-Dirac kernel $D_{W}(a \mu)$ to define the corresponding overlap Dirac matrix at nonzero density. The resultant overlap fermion action indeed has no $a^{-2}$ divergences $[9,10]$ in the free case. Unfortunately, however, it has no chiral invariance for nonzero $\mu$ either [10]. Using the definition of the chiral projectors for overlap fermions, we [11] proposed a chirally invariant Overlap action for nonzero $\mu$ :

$$
S^{F}=\sum_{n}\left[\bar{\psi}_{n, L}\left(a D_{o v}+a \mu \gamma^{4}\right) \psi_{n, L}+\bar{\psi}_{n, R}\left(a D_{o v}+a \mu \gamma^{4}\right) \psi_{n, R}\right]
$$




$$
=\sum_{n} \bar{\psi}_{n}\left[a D_{o v}+a \mu \gamma^{4}\left(1-a D_{o v} / 2\right)\right] \psi_{n} .
$$

It is easy to check that

- the action is indeed invariant under the chiral transformations, $\delta \psi=i \alpha \gamma_{5}\left(1-a D_{o v}\right) \psi$ and $\delta \bar{\psi}=i \alpha \bar{\psi} \gamma_{5}$, for any value of $a \mu$ or $a$.

- it reproduces the continuum action in the limit $a \rightarrow 0$ under $a \mu \rightarrow a \mu / M$ scaling, $M$ being the irrelevant parameter in overlap action.

- the chiral order parameter exists for all $\mu$ and $T$. It is defined by

$$
\langle\bar{\psi} \psi\rangle=\lim _{a m \rightarrow 0} \lim _{V \rightarrow \infty}\left\langle\operatorname{Tr} \frac{\left(1-a D_{o v} / 2\right)}{\left[a D_{o v}+\left(a m+a \mu \gamma^{4}\right)\left(1-a D_{o v} / 2\right)\right]}\right\rangle .
$$

It, however, has the same $\mu$-dependent $a^{-2}$ divergences in the number density and the energy density as the linear $\mu$-case for naive/staggered fermions [12]. Furthermore, unlike for the latter fermions, these cannot be removed [12] by exponentiation of the $\mu$-term. This leads us to a dilemma in case of the Overlap fermions: one either one sacrifices exact chiral invariance on the lattice or have it with the divergences in the continuum limit of $a \rightarrow 0$.

\section{Tackling the Divergences}

Motivated thus by the desire to maintain exact chiral symmetry at a finite lattice spacing for any value of the chemical potential $\mu$ in order to have a well defined order parameter protected by the chiral symmetry as a function of $(\mu, T)$, we opted to explore whether the $\mu$-dependent $a^{-2}$ divergences can be tackled without adopting the popular route of change of the fermionic action using terms that vanish in the continuum limit.

As a first step we examined carefully the free dense quark gas in continuum. We found that contrary to the common belief, these divergences are not due to lattice artifacts. Indeed, the $\mu$ dependent divergences exist in the continuum theory as well when appropriate care is taken while manipulating divergent integrals. The lattice regulator simply makes it easy to spot them. Using a Pauli-Villars cut-off $\Lambda$ in the continuum theory, one can also show the presence of $\mu \Lambda^{2}$ term in number density easily [13].

We will sketch the argument here briefly, referring the reader to [13] for more details. The expression for the free quark number density can be easily derived to be

$$
n=\frac{2 i T}{V} \sum_{n} \int \frac{d^{3} p}{(2 \pi)^{3}} \frac{\left(\omega_{n}-i \mu\right)}{p^{2}+\left(\omega_{n}-i \mu\right)^{2}} \equiv \frac{2 i T}{V} \sum_{n} \int \frac{d^{3} p}{(2 \pi)^{3}} F\left(\omega_{n}, \mu, \vec{p}\right),
$$

where $p^{2}=p_{1}^{2}+p_{2}^{2}+p_{3}^{2}$. All the gamma matrices are Hermitian in our convention. $T=0$ and $\mu=0$ corresponds to the vacuum contribution which can be removed by subtracting $n(T=0, \mu=0)$. While this is identically zero, the corresponding subtraction for the energy density is actually $\propto \Lambda^{4}$.

Employing the usual contour method, but with a cut-off $\Lambda$ for all four momenta, one has in the $T \rightarrow 0$ limit but $\mu \neq 0$ the number density as,

$$
n=2 i \int \frac{d^{3} p}{(2 \pi)^{3}}\left[-i \Theta(\mu-p)-\left(\int_{2}+\int_{4}+\int_{1}\right) \frac{d \omega}{\pi} \frac{\omega}{p^{2}+\omega^{2}}\right] .
$$




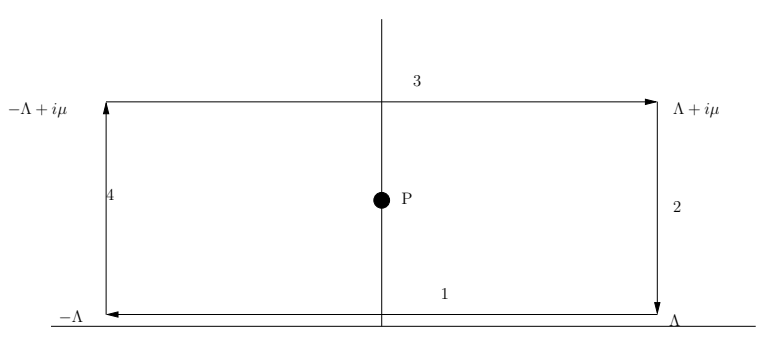

Figure 1: The contour diagram used for calculating the number density for free fermions in the continuum.

The corresponding contour diagram is shown in Figure 1.

Clearly the contribution of the arms 2 and 4 in the figure arises as extra terms due to the completion of the contour. The $\mu \Lambda^{2}$ terms can be shown to arise from the terms coming coming from these arms [13], being of the form $\log \left[\frac{p^{2}+(\Lambda+i \mu)^{2}}{p^{2}+(\Lambda-i \mu)^{2}}\right]$. If one lets $\Lambda \rightarrow \infty$, one may navely set the term to zero. A careful expansion in $\mu / \Lambda$ shows, however, that while the leading $\Lambda^{3}$ terms do cancel from the numerator and denominator, the $\mu \Lambda^{2}$ terms add together, and survive. Dropping the contribution from the arms 2 and 4, as is usually done, amounts to a subtracting the divergence by hand. One may follow this prescription of subtracting the free theory divergence by hand on the lattice as well. If it works, one can not only explore the QCD phase diagram with exact chiral fermions, but also have several computational advantages in computing the higher order susceptibilities needed in critical point search.

Indeed, for any fermion it leads to $M^{\prime}=\sum_{x, y} N(x, y)$, and $M^{\prime \prime}=M^{\prime \prime \prime}=M^{\prime \prime \prime \prime} \ldots=0$, in contrast to the $\exp ( \pm a \mu)$-prescription where all derivatives are nonzero: $M^{\prime}, M^{\prime \prime \prime} \ldots \neq 0$ and $M^{\prime \prime}, M^{\prime \prime \prime \prime}, M^{\prime \prime \prime \prime \prime \prime \prime} \ldots \neq$ 0 . As pointed out in [14], this results in a lot fewer terms in each of the Taylor coefficients, especially as the order increases. E.g., in the 4th order susceptibility, the dominant contribution to the diagonal part is due to the operator $\mathscr{O}_{4}=-6 \operatorname{Tr}\left(M^{-1} M^{\prime}\right)^{4}$ which has only a single term in the linear case compared to $\mathscr{O}_{4}=-6 \operatorname{Tr}\left(M^{-1} M^{\prime}\right)^{4}+12 \operatorname{Tr}\left(M^{-1} M^{\prime}\right)^{2} M^{-1} M^{\prime \prime}-3 \operatorname{Tr}\left(M^{-1} M^{\prime \prime}\right)^{2}-$ $3 \operatorname{Tr} M^{-1} M^{\prime} M^{-1} M^{\prime \prime \prime}+\operatorname{Tr} M^{-1} M^{\prime \prime \prime \prime}$ in the exponential (or generic $\left.f \dot{g}=1\right)$ case. The eighth order coefficient has a major contribution from $\mathscr{O}_{8}$. It too has one term for the linear case. To compute it one has to do eight sequential matrix inversions in contrast to 18 in the usual case. Moreover unlike in the linear method the $\mathscr{O}_{n}$ s have terms that occur with varying signs, these usually give rise to large fluctuations, and thus huge error bars, in determining the higher coefficients. Less cancellations may lead to better error estimate for the same CPU time and lesser number of $M^{-1}$ computations needed reduced the time even proportionately.

\section{Testing the idea}

We had earlier tested this proposal in the linear $\mu$ case with a simple subtraction of the free gas result on the $N_{t}=6$ full QCD $\left(N_{f}=2\right)$ configurations[15] by computing[16] all the coefficients and comparing them to the published results with the action exponential in $\mu$, i.e., without any subtraction. A good agreement within errors was observed. The Figure 2 displays the most precisely computed first coefficient $\chi_{20}$ normalized by its Stefan-Boltzmann value computed on the lattice of 


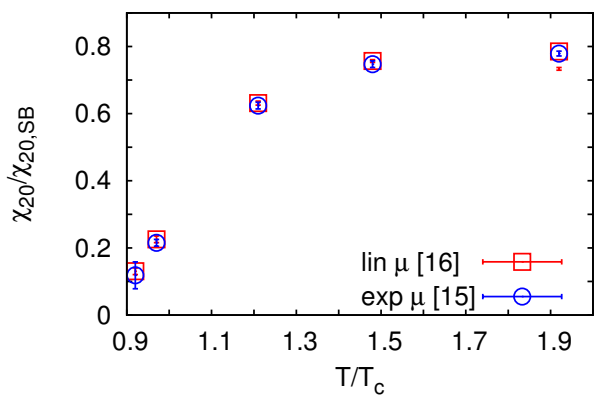

Figure 2: The ratio of the second order quark number susceptibility $\chi_{20}$ for two flavour QCD divided by the corresponding Stefan-Boltzmann value computed on the lattice of same size, compared at different temperatures for the linear and the exponential $\mu \operatorname{method}(\mathrm{GG})$.

same dimensions. It is natural to expect different finite size effects for different actions hence the results were normalized with the corresponding free fermion results for the $N_{t}=6$ case.

However, such a comparison falls short of testing the presence of any residual effects due to inadequate subtraction or even overcompensation since it is made at just one lattice spacing at each temperature. In order to test whether the divergence is truely absent, one needs to take the continuum limit $a \rightarrow 0$ or equivalently $N_{t} \rightarrow \infty$ at a fixed physical scale or temperature. Carrying out such a program for the full QCD is currently computationally hard. On the other hand, simulations for quenched QCD are easier done for larger $N_{t}$ needed for such an exercise. Considering the known empirical agreement for dimensionless ratios of physical quantities computed in quenched QCD and full QCD, such as the hadron spectrum or the pressure $\left(p / T^{4}\right)$ as a function of $T / T_{c}$, one expects the test in quenched QCD to be a good indication, in case the divergent effects are changed by interactions.

We therefore tested the idea for quenched QCD by employing $N_{t}=4,6,8,10$ and 12 lattices at two different temperatures, $T / T_{c}=1.25$ and 2 . These were chosen to exploit the known results for the critical couplings as a function of $N_{t}$ to avoid any fine-tuning in scale fixing. The results displayed below are for valence quark mass of $m / T_{c}=0.1$. The quark number susceptibility was measured on typically 50-100 independent configurations. The subtraction of the free gas divergent term was done for the respective $N_{t}$ in each case with the same values of valence quark mass. Absence of any divergent term in either case is evident from the positive slope of the data at both temperatures shown in Figure 3. Furthermore, the extrapolated continuum result coincides with the earlier result obtained with the $\exp ( \pm a \mu)$ action [17].

Since the valence quark mass introduces yet another scale a divergence, if present, could arise while reducing it. We lowered the mass by a factor of 10 to $m / T_{c}=0.01$ and repeated the exercise at the lower temperature, $T / T_{c}=1.25$. Once again the trend of the data in Figure 4 is same, and no divergent term is evidently present as inferred from the slope of the data. The comparison with the continuum extrapolated result with the exponential method was not feasible as the corresponding result for that action did not exist.

Finally, as a check of the scheme we also studied the next higher order susceptibility in a similar way. The fourth order susceptibility would have an additional unphysical $\mathscr{O}\left(a^{0}\right)$ term which 

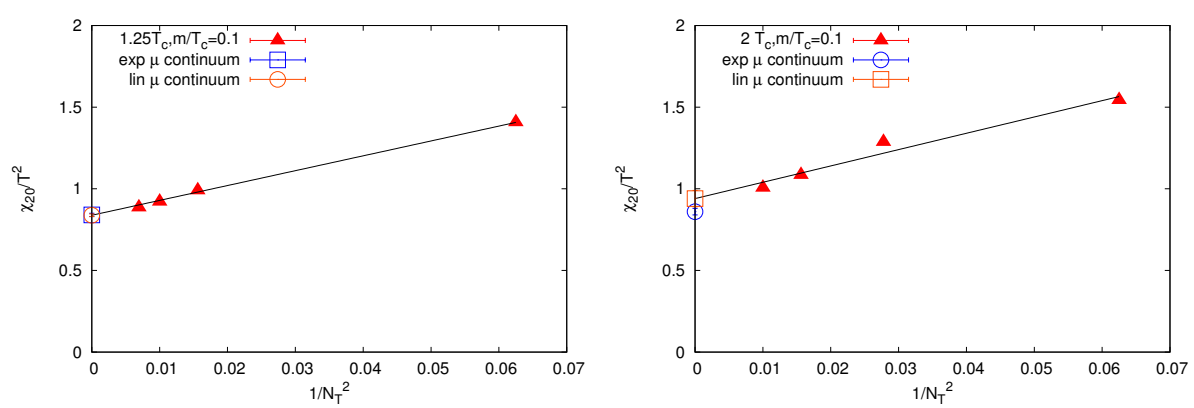

Figure 3: The continuum extrapolation for the second order susceptibility in quenched QCD at 1.25T (left panel) and $2 T_{c}$ (right panel) as a function of the lattice spacing or equivalently $N_{t}$ for $m / T_{c}=0.1$. The blue squares are the continuum results from the conventional method taken from Ref. [17].

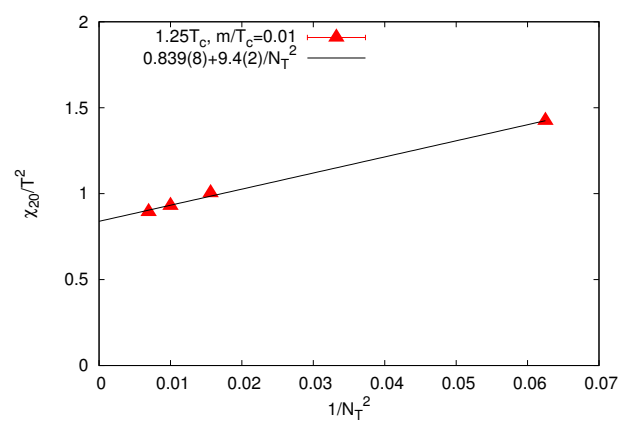

Figure 4: The continuum extrapolation for the second order susceptibility data in quenched QCD at $1.25 T_{c}$ for $m / T_{c}=0.01$.

we remove by subtracting the zero temperature part of the corresponding free fermions. It too shows similar finite result in continuum limit without any hints of any upward trend indicative of divergences. Moreover, a reasonable continuum limit is also visible by extrapolation.

\section{Summary}

In two recent studies $[13,16]$, we revisited the idea of introducing $\mu$ as a linear $(\mu N)$ term on the lattice, where $N$ is the conserved charge on the lattice. It is known to lead to $\mu$-dependent quadratic divergences in the number/energy density. Understanding the nature and origin of these divergences is both theoretically important as well as computationally advantageous since a suitable methodÂă of their elimination can allow for its much wider applicability including for exact chiral fermions.

We show here that the divergent $1 / a^{2}$ term in the second order susceptibility for free fermions is not a mere lattice artifact but it is present even in the continuum. Following the practice there, it can be subtracted off on the lattic. Since $N$ is a conserved charge, it does not get renormalized in the interacting theory. This implies that the coefficients of the divergent $1 / a^{2}$ term in full QCD should be the same as for the free fermions. We show that subtraction of such a free fermion 

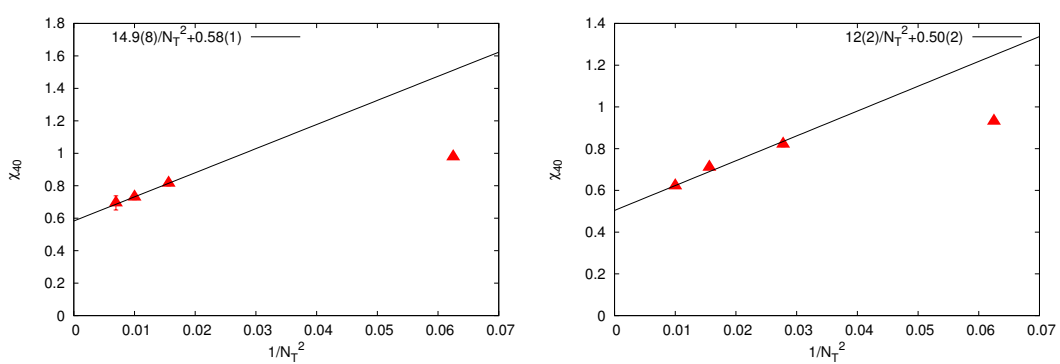

Figure 5: The continuum extrapolation for the fourth order diagonal susceptibility in quenched QCD at $1.25 T_{c}$ (left panel) and $2 T_{c}$ (right panel) as a function of the lattice spacing or equivalently $N_{t}$ for $m / T_{c}=0.1$.

term from the susceptibility in the (quenched) interacting case leaves only a physical piece. It has no divergences in the continuum limit and its extrapolation to continuum limit agrees with that computed by analytically eliminating the divergence. As in perturbation theory, once the zero temperature divergence is removed, interactions do not induce any additional divergence at finite $T$ or $\mu$ holds true non-perturbatively as well. We conclude that actions linear in $\mu$ can be employed safely with added computational advantages in computing the known fourth and higher order quark number susceptibilities, as well as yet higher order ones.

\section{References}

[1] P. Hasenfratz and F. Karsch Phys. Lett. B125, 308 (1983).

[2] J. Kogut et al., Nucl. Phys. B 225, 93 (1983).

[3] N. Bilic and R. V. Gavai Z. Phys. C23, 77 (1984).

[4] R. V. Gavai, Phys. Rev. D32, 519 (1985).

[5] R. V. Gavai and S. Gupta, Phys. Rev. D67, 034501 (2003).

[6] P. Hasenfratz, V. Laliena and F. Neidermeyer, Phys. Lett. B 427, 125 (1998).

[7] M. Luscher, Phys. Lett. B 428, 342 (1998).

[8] J. Bloch and T. Wettig, Phys. Rev. Lett. 97, 012003 (2006);

J. Bloch and T. Wettig, Phys. Rev. D76, 114511 (2007).

[9] C. Gattringer and L. Liptak, Phys. Rev. D76, 054502 (2007).

[10] D. Banerjee, R. V. Gavai and S. Sharma, Phys. Rev. D78, 014506 (2008);

D. Banerjee, R. V. Gavai and S. Sharma, PoS (LATTICE 2008), 177.

[11] R. V. Gavai and S. Sharma, Phys. Lett. B 716, 446 (2012).

[12] R. Narayanan and S. Sharma, JHEP 1110, 151 (2011);

[13] R. V. Gavai and S. Sharma, arXiv:1406.0474 [hep-lat].

[14] R. V. Gavai and S. Sharma, Phys. Rev. D81, 034501 (2010).

[15] R. V. Gavai and S. Gupta, Phys. Rev. D78, 114503 (2008).

[16] R. V. Gavai and S. Sharma, Phys. Rev. D85, 054508 (2012).

[17] S. Mukherjee, Phys. Rev. D74, 054508 (2006). 